\title{
P300 Responses Classification Improvement in Tactile BCI with Touch-sense Glove
}

\author{
Hiroki Yajima*, Shoji Makino*, and Tomasz M. Rutkowski* ${ }^{*} \dagger, \nabla$ \\ *Department of Computer Science and Life Science Center of TARA, University of Tsukuba, Japan \\ E-mail: tomek@bci-lab.info ( $\nabla$ corresponding author) \\ ${ }^{\dagger}$ RIKEN Brain Science Institute, Japan
}

\begin{abstract}
This paper reports on a project aiming to confirm whether a tactile stimulator "touch-sense glove" is effective for a novel brain-computer interface (BCI) paradigm and whether the tactile stimulus delivered to the fingers could be utilized to evoke event related potential (ERP) responses with possible attentional modulation. The tactile ERPs are expected to improve the BCI accuracy. The proposed new stimulator device is presented in detail together with psychophysical and EEG BCI experiment protocols. Results supporting the proposed "touch-sense glove" device are presented in form of online BCI classification accuracy results. Finally, we outline the future possible paradigm improvements.
\end{abstract}

\section{INTRODUCTION}

Many researchers recently join various projects related to a brain computer interface (BCI) technology which is expected to allow operation of any device using brainwaves only [1]. This technology shall allow disable people, e.g. the amyotrophic lateral sclerosis (ALS) users, to operate devices without any muscle activity necessary. The most popular $\mathrm{BCI}$ is a visual one in which user's control commands are estimated only from presented intentional responses to visual stimulus [1], [2], [3]. This modality, however, prevents users from paying attention to surrounding environment causing often difficulties in an application operation. Such BCI is not available also for users suffering from lost or bad vision [4]. Our research project proposes to use tactile BCI (tBCI) modality. This modality shall derive $\mathrm{P} 300$ response which is usually obtained by attending to a specific and known target [1] Although the auditory modality [5], [6], which is also an alternative to the vision, could also derive the $\mathrm{P} 300$ responses, it could not be used in case of advanced ALS patients (e.g. totally-locked-in syndrome) [6], [4]. Our research aims to improve tBCI classification accuracy and to develop a practical stimulation device. We search for the most suitable patterns leading to a successful multi command tactile paradigm.

The tBCI paradigm often utilizes a somatosensory steadystate response (SSSR) [7]. While a person is stimulated, the brain generates a response which has nearly the same frequency as the stimulus. The SSSR response becomes stronger when a person attends to the stimulus [7]. However, the SSSR does not appear when the stimulation period is very short, thus it is very difficult to gain a good information transfer rate (ITR) [1]. Recently BCI which utilizes an event related potential (ERP) instead of the SSSR has has been actively researched [6], [2], [8], [3], [4]. The ERP features are also

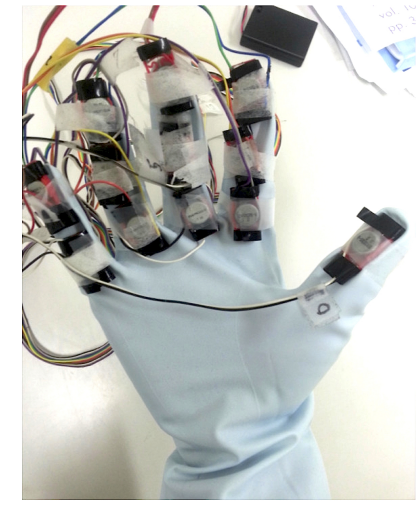

(a) Touch-sense glove

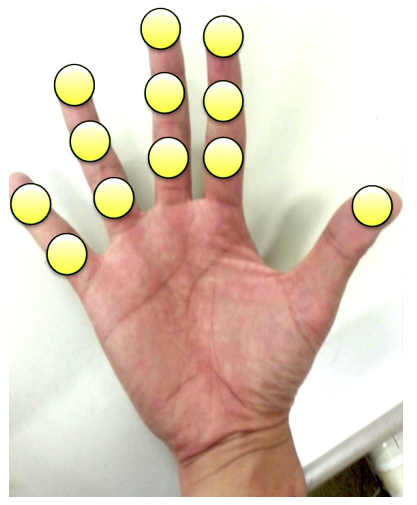

(b) Exciter positions
Fig. 1. Panel (a) presents the touch-sense glove on a used hand with 12 vibrotactile exciters attached along the fingers. Panel (b) presents detailed locations on a hand of the 12 vibrotactile exciters.

very suitable to classify a so called "aha-response" (known also as a P300 response since it is a positive ERP deflection after about $300 \mathrm{~ms}$ from the stimulus onset [1]). So far, the most popular tBCIs use fingertip stimulation to evoke P300 responses [9]. However, this modality's accuracy is still too low for practical utilization, thus, we propose to expand conventional stimulation to the whole finger surfaces.

The rest of the paper is organized as follows. In the next section we introduce methods used and developed within the project. Next, the obtained results and a discussion are presented. Conclusions and a future research direction outline summarize the paper.

\section{Methods}

In this section, we explain details of conventional and our proposed tBCI paradigms. In our novel tactile BCI paradigm project we conducted psychophysical and EEG experiments in order to compare new results with conventional methods. The psychophysical and EEG experiments were conducted in agreement with the ethical committee guidelines of the Faculty of Engineering, Information and Systems at University of Tsukuba, Tsukuba, Japan (experimental permission no. 2013R7). Five volunteer users participated in the experiments. We propose to utilize $\mathrm{P} 300$ response in the tBCI paradigm and a classification method based on a classical 


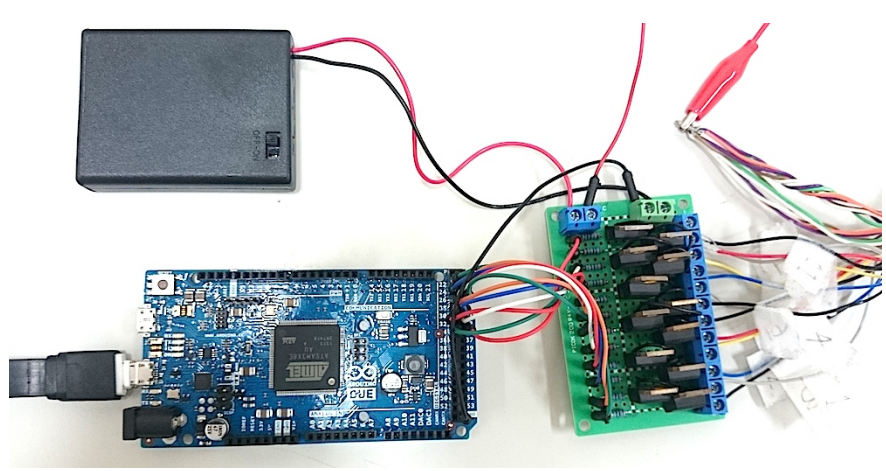

Fig. 2. ARDUINO DUE micro-controller board with output ports connected to our own design $12-$ channels amplifier. The vibrotactile exciters are connected to the amplifier outputs and powered for security from a black battery pack in the upper part of the photograph. The communication with our experiment managing MAX 6 visual programming environment patch is established via an USB port with RS232 serial communication embedded protocol.

oddball paradigm [1]. The $\mathrm{P} 300$ response is a positive deflection starting at around $300 \mathrm{~ms}$ after the user attends stimulus and it does not appear to the ignored one [1]. Usually the $\mathrm{P} 300$ responses are applied to visual and auditory $\mathrm{BCI}$ modalities [2]. The P300-based BCI discriminates the attended and ignored stimuli from the differences in ERP shapes. An example of the averaged P300 response evoked to an expected target stimuli in comparison to the ignored non-targets is presented in Figure 5. In our experiments, we assign label to the attended stimulus as the target and to the other ignored as the non-target.

We proposed a new stimulator made of a glove which we named "a touch-sense glove" as shown in Figure 1(a). The glove had embedded 12 vibrotactile exciters which were attached to user finger are as depicted also in Figure 1(a). A detailed placement of the exciter positions is outlined in Figure 1 . The reason why the vibrotactile excites were attached to a glove was to enhance convenience of an experimental setup avoiding manual attachment of the 12 devices separately each time. A rubber glove served also as a safety electric insulator to avoid any current leakage causing a possible electric interference with EEG.

We first conducted psychophysical experiments in order to determine the task difficulties based on the recorded behavioral "button press" responses which were executed after the identified stimulus patterns. After that, in order to evaluate the P300

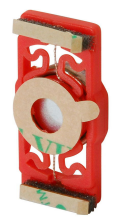

(a) Front side attached to fingers.

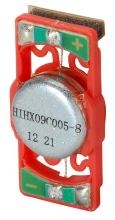

(b) Back side.
Fig. 3. Tactile exciter HIHX09C005-8 by HiWave used in psychophysical and EEG experiments.
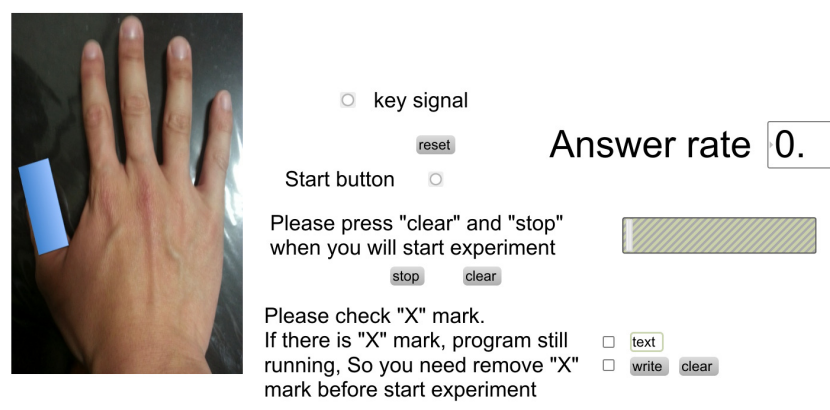

Fig. 4. The psychophysical experiment instruction screen. The blue rectangle is a target stimulus pattern. A user responses are collected using a computer keyboard button-press answers. Correct answer rates are displayed after each trial to update the user with accuracy results.

response occurrences and a possible online BCI application based on online classification, we conducted EEG experiments with the same users as in the previous psychophysical experiment.

\section{A. Experimental Device Details}

The ARDUINO DUE micro-controller board was used to generate square wave signals delivered to vibrotactile exciters as presented in Figure 2. The control of the ARDUINO DUE board was based on a simple program communicating with a portable computer via an USB port with the RS232 serial communication protocol embedded. The serial communication with ARDUINO DUE board was managed by a MAX 6 program developed by our team. The vibrotactile exciters (see Figure 3) used in the experiments were attached to ARDUINO DUE via a custom made multichannel amplifier developed in our laboratory as shown in Figure 2. In psychophysical and EEG experiments, there were five stimulus patterns as shown in Table I. The stimulus patterns in form of vibrotactile exciter

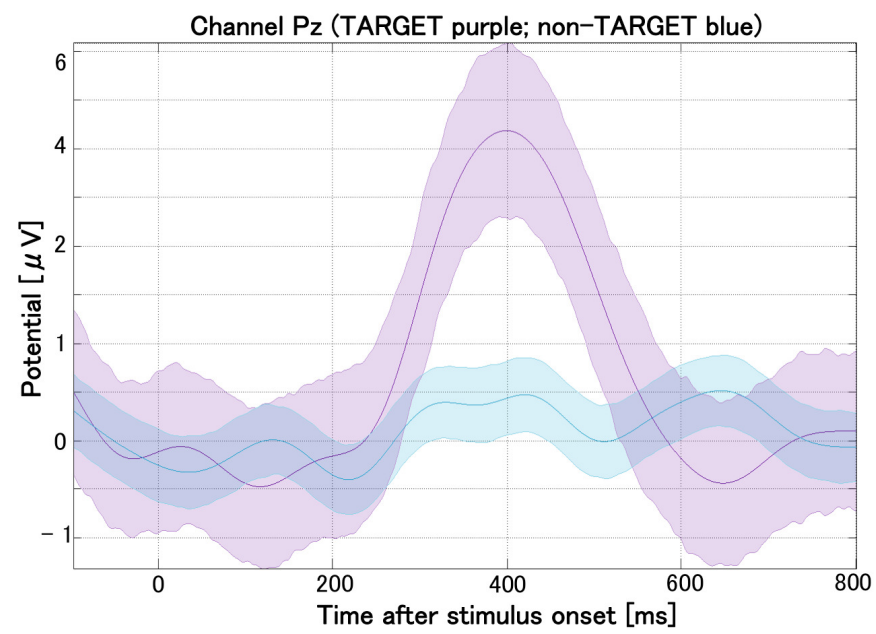

Fig. 5. This figure presents the $\mathrm{P} 300$ response which is the positive deflection after $300 \mathrm{~ms}$ from the stimulus onset. The purple line represents the averaged response to the target stimuli, while the blue to the non-targets. 


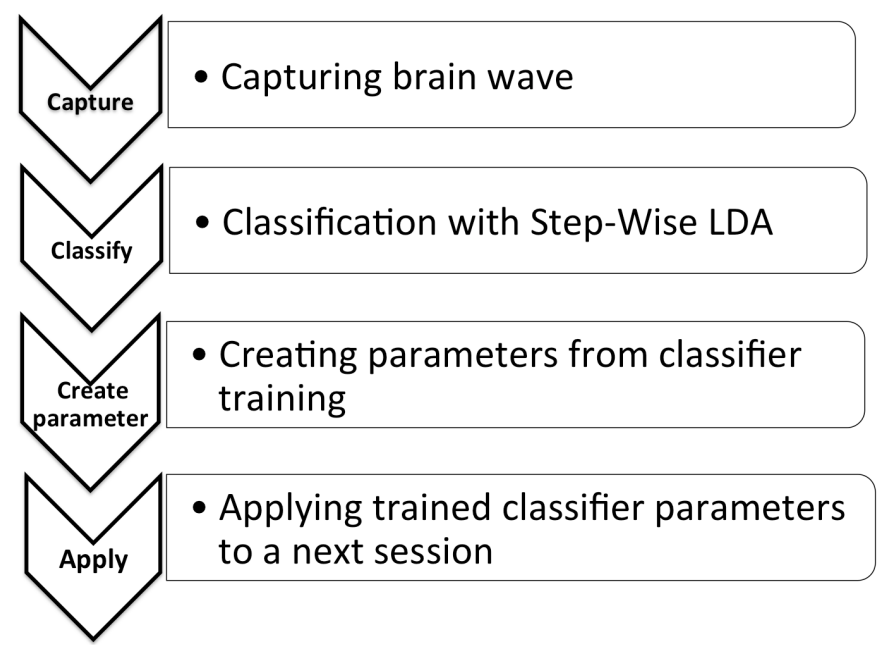

Fig. 6. A flow diagram of EEG signal processing and brainwaves classifier training procedures.

TABLE I

FINGER STIMULUS PATTERN DETAILS USING VIBROTACTILE EXCITERS

\begin{tabular}{|r|c|c|c|c|c|}
\hline Stimulus number & 1 & 2 & 3 & 4 & 5 \\
\hline Stimulus type & thumb & forefinger & $\begin{array}{c}\text { middle } \\
\text { finger }\end{array}$ & $\begin{array}{c}\text { ring } \\
\text { finger }\end{array}$ & $\begin{array}{c}\text { little } \\
\text { finger }\end{array}$ \\
\hline Number of exciters & 1 & 3 & 3 & 3 & 2 \\
\hline
\end{tabular}

sets vibrated for $100 \mathrm{~ms}$. Each stimulus instruction pattern was represented on a user interface display by a blue rectangle as shown in Figure 4.

\section{B. Psychophysical Experiment Protocol}

Before the EEG experiments, we conducted the psychophysical study to check whether the users can distinguish tactile apparent motion stimulus patterns. In the psychophysical experiments, user button press responses were recorded. Based on the recorded response datasets, we analyzed the correct answer rates and the response times. All the psychophysical experiment had the same protocol. The experimental procedure consisted of the following steps:

TABLE II

THE PSYCHOPHYSICAL EXPERIMENT CONDITIONS

\begin{tabular}{|r|l|}
\hline Condition & Detail \\
\hline \hline Number of users & 5 \\
\hline Mean age & 26.6 \\
\hline Stimulus duration & $100 \mathrm{~ms}$ \\
\hline Stimulus frequency & $300 \mathrm{~Hz}$ \\
\hline Inter-stimulus-interval (ISI) & $500 \mathrm{~ms}$ \\
\hline Stimulus device & 12 vibrotactile exciters \\
\hline Number of sessions for each user & 2 \\
\hline Number of trials in each run & $\begin{array}{l}5(50 \text { targets } \\
\text { and } 200 \text { non-targets) }\end{array}$ \\
\hline
\end{tabular}

TABLE III

THE EEG EXPERIMENT CONDITIONS

\begin{tabular}{|c|c|}
\hline Condition & Detail \\
\hline Number of users & 5 \\
\hline Mean age & 26.6 \\
\hline Stimulus duration & $100 \mathrm{~ms}$ \\
\hline Inter-stimulus-interval (ISI) & $500 \mathrm{~ms}$ \\
\hline Stimulus device & 12 HIHX09C005-8 exciters \\
\hline Number of stimuli & $\begin{array}{l}5 \text { (thumb; forefinger; middle finger; } \\
\text { ring finger; little finger) }\end{array}$ \\
\hline EEG recording system & $\begin{array}{l}\text { g.USBamp amplifier and active wet } \\
\text { EEG electrodes system }\end{array}$ \\
\hline Number of the EEG channels & 8 \\
\hline EEG electrode positions & $\begin{array}{l}\mathrm{Cz}, \mathrm{CPz}, \mathrm{P} 3, \mathrm{P} 4, \mathrm{C} 3, \mathrm{C} 4, \\
\mathrm{CP} 5 \text {, and } \mathrm{CP} 6\end{array}$ \\
\hline Sampling rate & 512 \\
\hline high-pass; low-pass; notch & $0.1 \mathrm{~Hz} ; 60 \mathrm{~Hz} ; 48 \sim 52 \mathrm{~Hz}$ \\
\hline Reference electrode & Behind the user's left ear \\
\hline Ground electrode & On a forehead $(\mathrm{FPz})$ \\
\hline Number of sessions & 3 \\
\hline Number of trials in each run & 5 (50 targets and 200 non-targets) \\
\hline
\end{tabular}

1) the visual instruction on a computer display and a stimulus to the user finger were given of which pattern to attend next;

2) a sequence of tactile stimuli was delivered to the fingers (an oddball sequence of random ordered patterns);

3) the user paid attention only to the instructed pattern (the target) while ignoring the others;

4) the above three steps were repeated until all the stimulus patterns become the targets.

The above four steps defined a single sequence. We conducted the five trials for psychophysical experiments because of the five different target stimulus patterns used. We assigned all sequences to a single session and we conducted two experimental sessions for each user. A single trial was composed of 50 randomized order stimuli which consisted the ten targets and forty non-targets. If the number of button-presses to each trial was less than the designed number, we did treat it as a no response of an overlooking condition. Each trial consisted of the randomized order presentations with fixed inter-stimulus-interval (ISI) and the stimulus durations. All psychophysical experiments were conducted by a personal computer running MAX 6 program with the same generated outputs. The same MAX 6 program registered the behavioral button-press response times and stimulus numbers to which the user responded. The stimulus patterns have been summarized in Table I and detailed summary of the psychophysical experiment conditions is presented in Table II. The user was instructed to attend to the target pattern presented in advance before each random stimulus sequences. The user instruction of which pattern to attend was delivered on a computer screen as presented in Figure 5. The instruction with the target 
displayed was changing after each trial. At each trial, the user could confirm the answer rate success of the executed buttonpresses. The user generated the behavioral responses with a free second hand.

\section{EEG Experiment Protocol}

In order to evaluate the P300 response occurrences and the online BCI classification accuracies, we conducted a series of EEG experiments with the same users as in the previously described psychophysical experiment section. The EEG experiments did not require the users to respond behaviorally by pressing a button, but only mental responses were instructed. Figure 6 presents a flow diagram of EEG signal processing and brainwaves classifier training pipeline. First, the user's brainwaves were captured using wet active EEG electrodes. Second, the captured and filtered brain signals were segmented and classified after a training of the step-wise linear discriminant analysis (SWLDA) classifier [10]. Next, the parameters of the trained SWLDA classifier were entered to the BCI2000 software [11] for the subsequent online BCI sessions. Each BCI command output was a result of features drawn from the averaged responses and classified by the SWLDA method within the BCI2000 environment.

The EEG experimental procedure consisted of the the same steps as in the previous psychophysical experiments. A single EEG experiment BCI trial was composed of randomized 10 targets and 40 non-target stimuli. The EEG signals were captured with g.USBamp EEG amplifier by g.tec Medical Engineering, Austria, with 8 wet active electrodes g.LADYbird by the same manufacturer. The electrodes were attached to the following scalp locations $\mathrm{Cz}, \mathrm{CPz}, \mathrm{P} 3, \mathrm{P} 4, \mathrm{C} 3, \mathrm{C} 4, \mathrm{CP} 5$, and CP6. The ground was attached to the Fpz and a reference electrode on a left earlobe. The recorded EEG signals were processed by BCI2000 application [11], using the SWLDA classifier [10]. The sampling rate was set to $512 \mathrm{~Hz}$. A notch filter to remove power line interferences was set at a rejection band of $48 \sim 52 \mathrm{~Hz}$. Next, the EEG signals were digitally bandpass processed be high-pass and low-pass filters set at $0.1 \mathrm{~Hz}$ and $40.0 \mathrm{~Hz}$ respectively. A procedure of 10 single ERP responses averaging was used in order to enhance the P300 amplitudes. In EEG experiment, we also presented the same instruction screen as shown in Figure 4. P300 (the user intentions) classification results were presented in form of numeric values using the same instruction screen. The stimulus patterns and vibration times have been summarized in Table II. The EEG recording detailed conditions have been summarized in Table III. In the EEG experiment, we conducted three runs for each user. The single run was composed of 50 targets and 200 non-targets. A single sequence was composed of 10 trials (10 targets and 40 non-targets presented randomly in the oddball paradigm). The user was instructed to attend to the target patterns presented in advance before each oddball sequence.

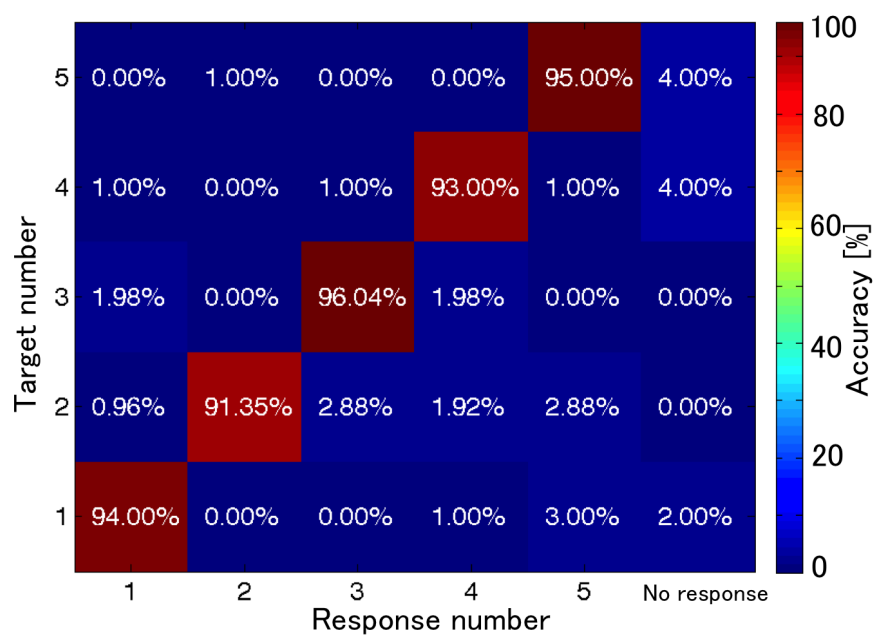

Fig. 7. The psychophysical experiment results in form of a confusion matrix of the grand mean averaged user accuracy results. The horizontal axis represents user response numbers and the vertical the instructed targets respectively. A "no response" column indicates the missed responses by the users. A diagonal indicates the correct responses, while off diagonals the mistakes. The obtained accuracy values are also presented with color coding.

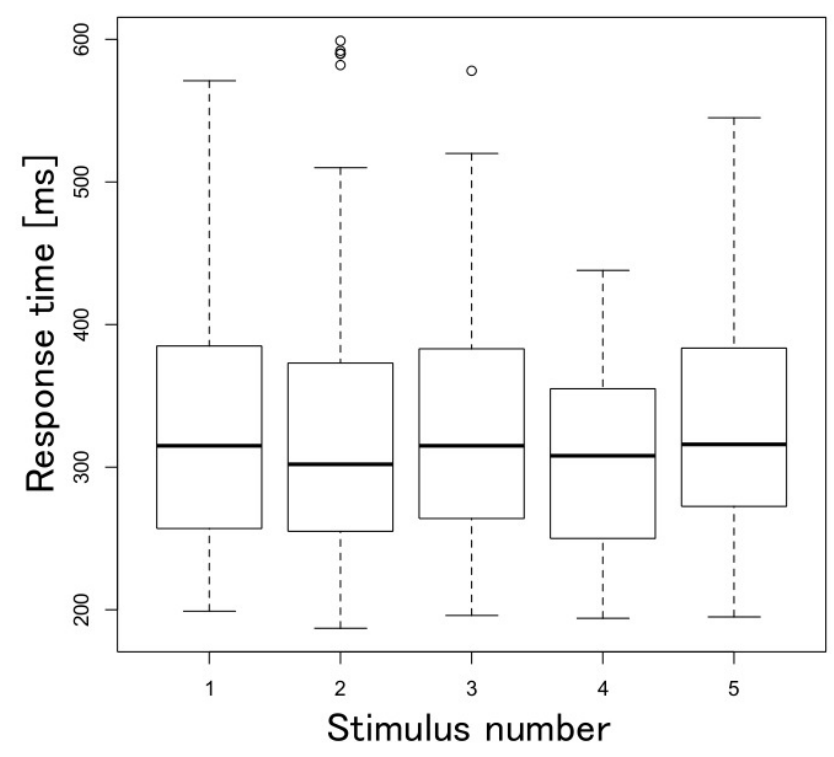

Fig. 8. The psychophysical experiment results in form of boxplots of the grand mean averaged user response times (behavioral button-presses). The horizontal axis represents the stimulus numbers and the vertical one the response times, respectively. No significant differences among the stimulus patterns were observed.

\section{RESUlts}

In this section, we present results of the two experimental sessions conducted, namely the psychophysical and online BCI EEG studies outcomes. 
$\mathrm{Cz}$

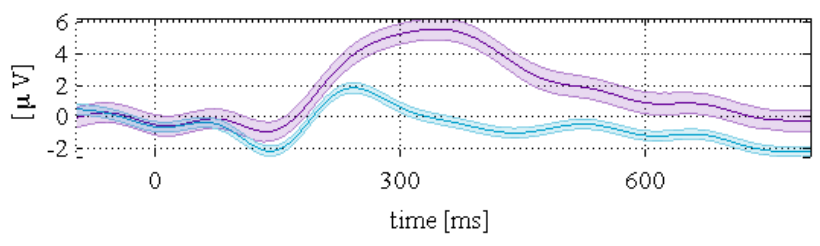

P3

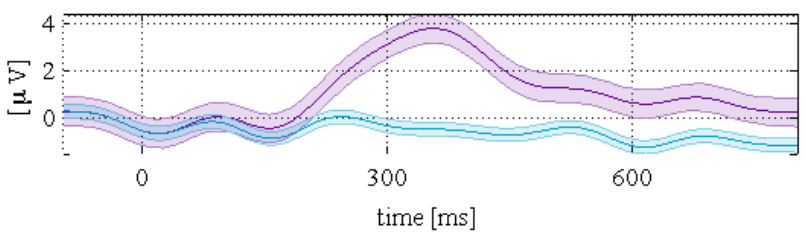

C3

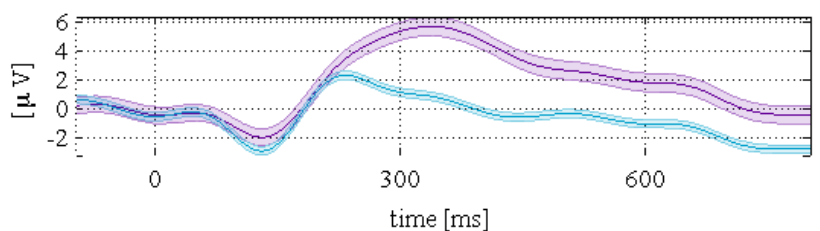

CP5

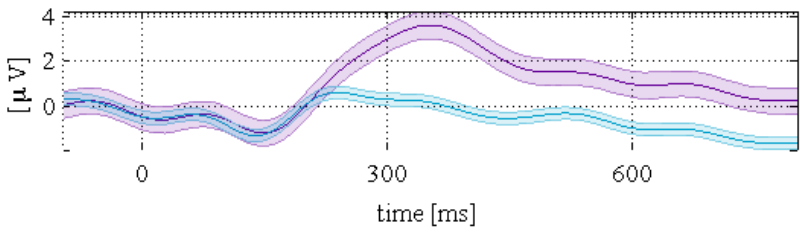

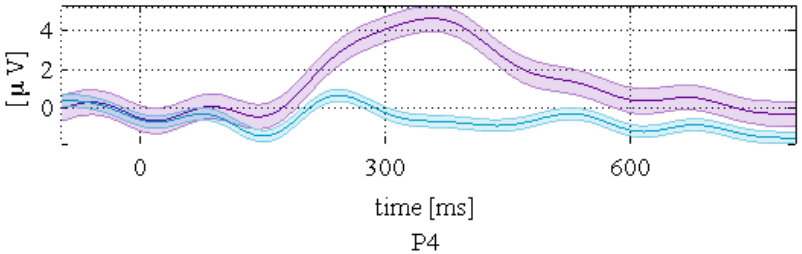

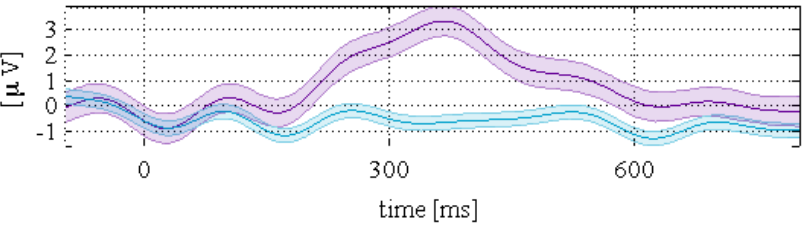

C4

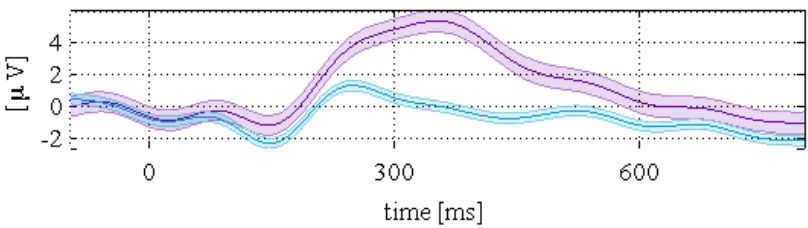

CP6

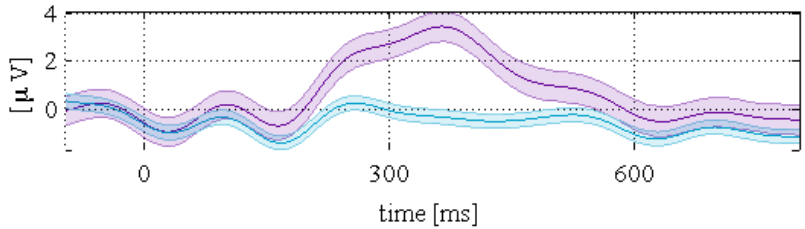

Fig. 9. Grand mean ERP averaged results up to $800 \mathrm{~ms}$ from the first EEG experiment. Each channel panel presents the averaged ERP plot of the TARGET and non-TARGET. The purple lines depict TARGETs and blue non-TARGETs respectively. The P300 peaks seem to be in the latency the range of $200 \sim 600$ ms.

\section{A. Psychophysical Experiment Results}

As a result of the conducted psychophysical experiments we obtained user response accuracies and reaction times. A confusion matrix depicted in Figure 7 was generated based on the averaged response accuracies of the all five users who took part in the psychophysical experiments. A horizontal axis in in Figure 7 represents the stimulus numbers, while the vertical one the button-press (behavioral) response times. A "no response" column has been included to represent the omitted responses. A diagonal line of the confusion matrix represents correct responses. A color coding has been used additional to visualize graphically psychophysical experiment accuracies. The resulted accuracies were above $90 \%$ level, which was evaluated as a very good outcome and way above a chance level of $20 \%$. As the result of the psychophysical experiment we confirmed the users in our experiments could distinguish all five vibrotacltile stimulus patterns delivered using the proposed touch-sense glove. Figure 8 reports response time distributions in form of boxplots. The horizontal axis in this figure represent the stimulus numbers, while the vertical one the button-press (behavioral) response times in milliseconds. This boxplot shows that the users reacted to target stimuli with median time of about $300 \mathrm{~ms}$. No significant differences were observed among the response times to various patterns as tested with ANOVA.
TABLE IV

THE EEG EXPERIMENT BCI CLASSIFICATION (THEORETICAL CHANCE LEVEL OF 20\%) AND ITR SCORES (THE AVERAGED BEST ITR WAS 3.94)

\begin{tabular}{|c|c|c|c|}
\hline user number & $\begin{array}{c}\text { The best classification } \\
\text { accuracy }\end{array}$ & $\begin{array}{c}\text { Averaged } \\
\text { accuracy }\end{array}$ & The best ITR \\
\hline \hline 1 & $100 \%$ & $100 \%$ & 4.64 \\
\hline 2 & $100 \%$ & $90 \%$ & 4.64 \\
\hline 3 & $100 \%$ & $60 \%$ & 4.64 \\
\hline 4 & $100 \%$ & $70 \%$ & 4.64 \\
\hline 5 & $60 \%$ & $40 \%$ & 1.10 \\
\hline
\end{tabular}

\section{B. EEG Experiment Results}

In this section, we present the online BCI EEG experiment results. The brainwaves have been depicted in form of grand mean averaged (all users and sessions) ERPs in Figure 9 and as the area under the curve (AUC) of the EEG features discrimination analysis results with head topographic plots in Figure 10. The above results clearly indicated P300 response validity and potential separability as indicated by AUC values above 0.5 benchmarks in Figure 10 in the latencies of $250 \sim$ $550 \mathrm{~ms}$. Online tBCI experiments results are also summarized in form user achieved accuracies (a chance level was of $20 \%$ ) and information transfer rates, which were calculated 

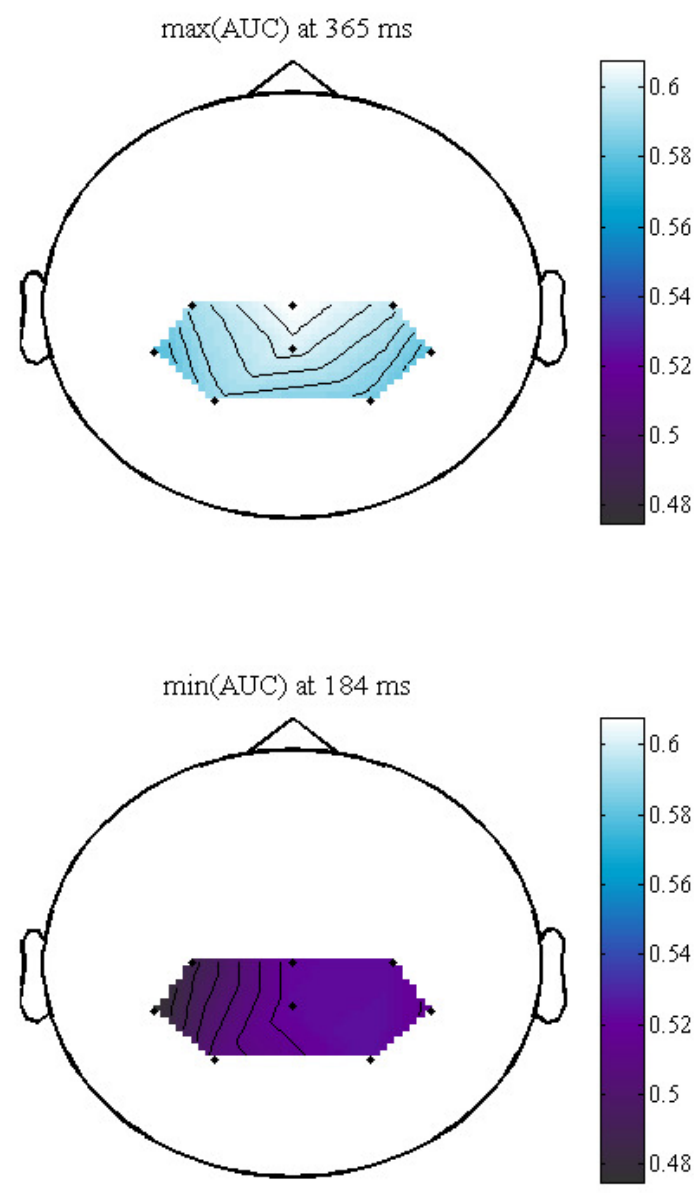

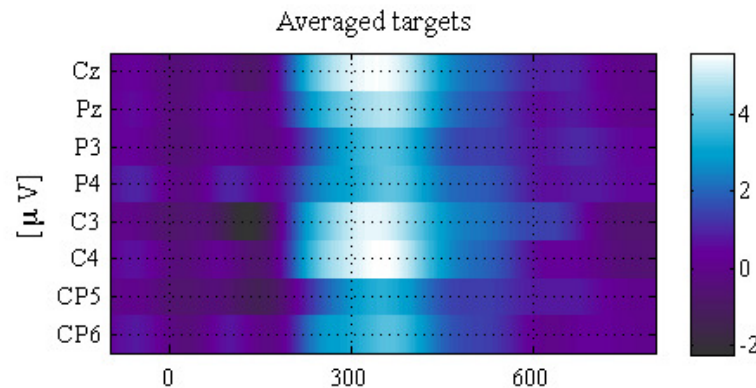

Averaged non-targets

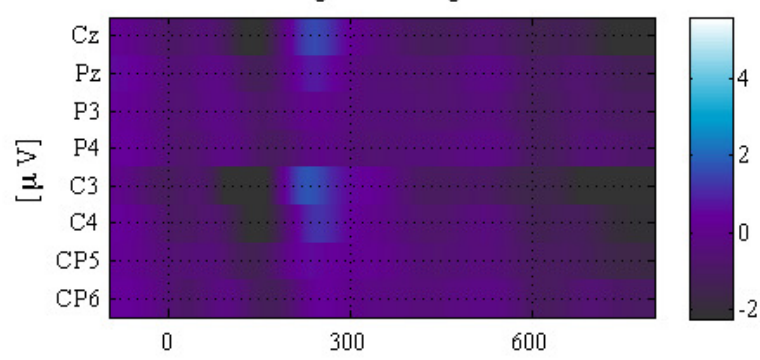

Targets vs. non-targets AUC scores

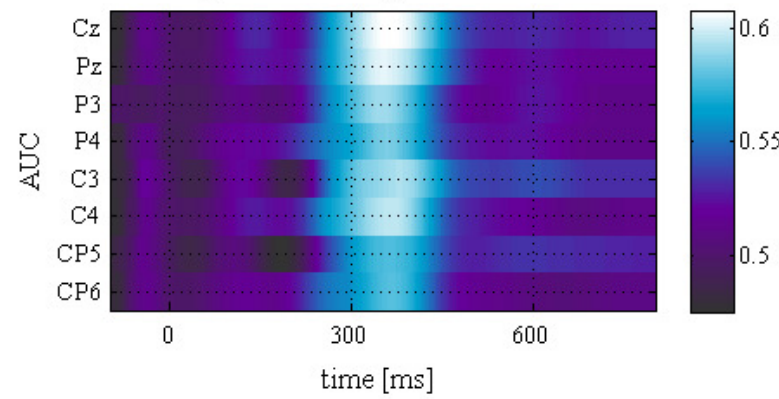

Fig. 10. Grand mean AUC averaged results from the first EEG experiment. The top head topographic plots present the AUC scores spatial distributions and the maximum (here $365 \mathrm{~ms}$ ) and minimum (here $184 \mathrm{~ms}$ ) latencies. The second and third from the top matrix plots depict the TARGET and non-TARGET grand mean averaged responses for all EEG channels used in the experiment. The bottom panel presents the AUC scores matrix for TARGET versus non-TARGET distributions separability evaluation.

as follwos

$$
I T R=V \cdot R
$$

where $V$ was the classification speed in selections/min and $R$ the number of bits/selection calculated as,

$$
R=\log _{2} N+P \log _{2} P+(1-P) \log _{2} \frac{1-P}{N-1},
$$

with $N$ was a number of classes (five in this study) and $P$ the obtained in online BCI experiments classification accuracy. The accuracy and ITR results are listed in the Table IV. The averaged values reported in Table IV are based on arithmetic means of the brainwave classification accuracies. The best classification accuracy was the maximum accuracy the user could score in the whole experiment. The averaged accuracy was the mean from the two sessions each user conducted. We also presented the maximum accuracies with scores reaching $100 \%$ for four out of five users taking part in the study. The lowest averaged accuracy was of $40 \%$ which was still above the chance level of $20 \%$ in the presented study. The obtained
ITR results would allow for a slow yet already comfortable interaction using the proposed tBCI paradigm.

\section{CONCLUSIONS}

In this paper, the psychophysical and EEG experiments were conducted in order to confirm our research hypothesis of the novel stimulator named "the touch-sense glove" usability for the tBCI. In the series of psychophysical and EEG experiments we confirmed that the users could distinguish the five vibrotactile stimulus patterns delivered to the five fingers of the dominant hand. We could also observe clear and possible to discriminate brainwave $\mathrm{P} 300$ responses.

The tBCI concept was evaluated in online classification experiments with ten trails averaging setup using SWLDA classifier of the P300 responses resulting with the final commands.

The obtained results have shown that the averaged classification accuracies resulted above the chance level of $20 \%$. 
The online $\mathrm{tBCI}$ averaged accuracy results were in a range of $40 \% \sim 100 \%$. The best obtained ITR was of $4.64 \mathrm{bit} / \mathrm{min}$.

As a result of the conducted study, we could draw the following conclusions:

1) The results of the conducted psychophysical experiments showed that the users could distinguish the tactile stimulation generated by the proposed "touch-sense glove."

2) We could confirm the clear P300 responses in EEG experiments in which tactile stimulation was generated by the "touch-sense glove."

3) The EEG experiments resulted with the easily discriminable $\mathrm{P} 300$ responses leading to the classification accuracies and ITR scores above the chance levels, or even with perfect scores within the limitations of the experiential settings.

We plan to continue this line of research in the near future to conduct experiments with shorter ISIs and with single trialbased classification sequences.

\section{ACKNOWLEDGEMENTS}

This research was supported in part by the Strategic Information and Communications R\&D Promotion Program no. 121803027 of The Ministry of Internal Affairs and Communication in Japan, and by KAKENHI, the Japan Society for the Promotion of Science, grant no. 24243062.

\section{REFERENCES}

[1] J. Wolpaw and E. W. Wolpaw, Eds., Brain-Computer Interfaces: Principles and Practice. Oxford University Press, 2012.

[2] M. Chang, N. Nishikawa, Z. R. Struzik, K. Mori, S. Makino, D. Mandic, and T. M. Rutkowski, "Comparison of P300 responses in auditory, visual and audiovisual spatial speller BCI paradigms," in Proceedings of the Fifth International Brain-Computer Interface Meeting 2013. Asilomar Conference Center, Pacific Grove, CA USA: Graz University of Technology Publishing House, Austria, June 3-7, 2013, p. Article ID: 156. [Online]. Available: http://castor.tugraz.at/doku/BCIMeeting2013/156.pdf

[3] J. E. Huggins, C. Guger, B. Allison, C. W. Anderson, A. Batista, A.-M. Brouwer, C. Brunner, R. Chavarriaga, M. Fried-Oken, A. Gunduz, D. Gupta, A. Kübler, R. Leeb, F. Lotte, L. E. Miller, G. Müller-Putz, T. Rutkowski, M. Tangermann, and D. E. Thompson, "Workshops of the fifth international braincomputer interface meeting: Defining the future," Brain-Computer Interfaces, vol. 1, no. 1, pp. 27-49, 2014. [Online]. Available: http://www.tandfonline.com/doi/abs/10.1080/2326263X.2013.876724

[4] T. M. Rutkowski and H. Mori, "Tactile and bone-conduction auditory brain computer interface for vision and hearing impaired users," Journal of Neuroscience Methods, p. Available online 21 April 2014, 2014 [Online]. Available: http://dx.doi.org/10.1016/j.jneumeth.2014.04.010

[5] T. M. Rutkowski, A. Cichocki, and D. P. Mandic, "Spatial auditory paradigms for brain computer/machine interfacing," in International Workshop On The Principles and Applications of Spatial Hearing 2009 (IWPASH 2009) - Proceedings of the International Workshop, MiyagiZao Royal Hotel, Sendai, Japan, November 11-13, 2009, p. P5.

[6] Y. Matsumoto, N. Nishikawa, S. Makino, T. Yamada, and T. M. Rutkowski, "Auditory steady-state response stimuli based bci application-the optimization of the stimuli types and lengths," pp. $1-7,2012$.

[7] G. Muller-Putz, R. Scherer, C. Neuper, and G. Pfurtscheller, "Steadystate somatosensory evoked potentials: suitable brain signals for braincomputer interfaces?" Neural Systems and Rehabilitation Engineering, IEEE Transactions on, vol. 14, no. 1, pp. 30-37, 2006.

[8] H. Mori, Y. Matsumoto, V. Kryssanov, E. Cooper, H. Ogawa, S. Makino, Z. R. Struzik, and T. M. Rutkowski, "Multi-command tactile brain computer interface: A feasibility study," in Haptic and Audio Interaction Design. Springer, 2013, pp. 50-59.
[9] H. Mori, Y. Matsumoto, S. Makino, V. Kryssanov, and T. M. Rutkowski, "Vibrotactile stimulus frequency optimization for the haptic BCI prototype," in Proceedings of The 6th International Conference on Soft Computing and Intelligent Systems, and The 13th International Symposium on Advanced Intelligent Systems, Kobe, Japan, November 20-24, 2012, pp. 2150-2153. [Online]. Available: http://arxiv.org/abs/1210.2942

[10] D. J. Krusienski, E. W. Sellers, F. Cabestaing, S. Bayoudh, D. J. McFarland, T. M. Vaughan, and J. R. Wolpaw, "A comparison of classification techniques for the P300 speller,' Journal of Neural Engineering, vol. 3, no. 4, p. 299, 2006. [Online]. Available: http://stacks.iop.org/1741-2552/3/i=4/a=007

[11] G. Schalk and J. Mellinger, Human-Computer Interaction: Practical Guide to Brain-Computer Interfacing with Bci2000: General-Purpose Software for Brain-Computer Interface Research, Data Acquisition, Stimulus Presentation, and Brain Monitoring. Springer, 2010. 